\title{
GPS-Optimal Micro Air Vehicle Navigation in Degraded Environments*
}

\author{
Jason T. Isaacs ${ }^{1}$, Ceridwen Magee ${ }^{2}$, Anantharaman Subbaraman ${ }^{1}$, François Quitin ${ }^{3}$, Kingsley Fregene ${ }^{2}$, \\ Andrew R. Teel ${ }^{1}$, Upamanyu Madhow ${ }^{1}$, and João P. Hespanha ${ }^{1}$
}

\begin{abstract}
We investigate a computationally and memory efficient algorithm for radio frequency (RF) source-seeking with a single-wing rotating micro air vehicle (MAV) operating in an urban canyon environment. We present an algorithm that overcomes two significant difficulties of operating in an urban canyon environment. First, Global Positioning System (GPS) localization quality can be degraded due to the lack of clear line of sight to a sufficient number of GPS satellites. Second, the spatial RF field is complex due to multipath reflections leading to multiple maxima and minima in received signal strength (RSS). High quality GPS localization is maintained by observing the GPS signal to noise ratio (SNR) to each satellite and making inferences about directions of high GPS visibility (allowable) and directions of low GPS visibility (forbidden). To avoid local maxima in RSS due to multipath reflections we exploit the rotation of the MAV and the directionality of its RF antenna to derive estimates of the angle of arrival (AOA) at each rotation. Under mild assumptions on the noise associated with the AOA measurements, a greedy algorithm is shown to exhibit a global recurrence property. Simulations supplied with actual GPS SNR measurements indicate that this algorithm reliably finds the $R F$ source while maintaining an acceptable level of GPS visibility. Additionally, outdoor experiments using Lockheed Martin's Samarai MAV demonstrate the efficacy of this approach for static source-seeking in an urban canyon environment.
\end{abstract}

\section{INTRODUCTION}

Unmanned Aircraft Systems (UAS) are becoming prevalent in many civilian and military applications such as surveillance, weather monitoring and critical infrastructure inspection. Due to their lower size, weight, required power, cost and ease of deployment/maintenance, the class of UAS that will be most widely deployed will be the portable (small or micro) UAS that typically weigh no more than a few pounds (see [1] for a recent classification of UAS based on gross weight and flight endurance). Of this group, rotary wing UAS are particularly attractive in applications where an ability to hover, takeoff/land vertically and/or operate at

*This work was supported by the Institute for Collaborative Biotechnologies through grant W911NF-09-0001 from the U.S. Army Research Office, grant FA9550-12-1-0127 from AFOSR, and grant ECCS-1232035 from the NSF. The content of the information does not necessarily reflect the position or the policy of the Government, and no official endorsement should be inferred.

${ }^{1}$ J.T. Isaacs, A. Subbaraman, A.R. Teel, U. Madhow, and J.P. Hespanha are with the Department of Electrical and Computer Engineering, University of California Santa Barbara, CA 93106 \{jtisaacs, teel, madhow, hespanha\}@ece.ucsb.edu, anantharamaneumail.ucsb.edu

${ }^{2} \mathrm{C}$. Magee and K. Fregene are with the Lockheed Martin Advanced Technologies Laboratories, Cherry Hill, NJ 08002 \{ceridwen.magee, kingsley.fregene\} a lmco.com

${ }^{3}$ F. Quitin is with the School of EEE, Nanyang Technological University, Singapore 639798 fquitin@ntu.edu.sg

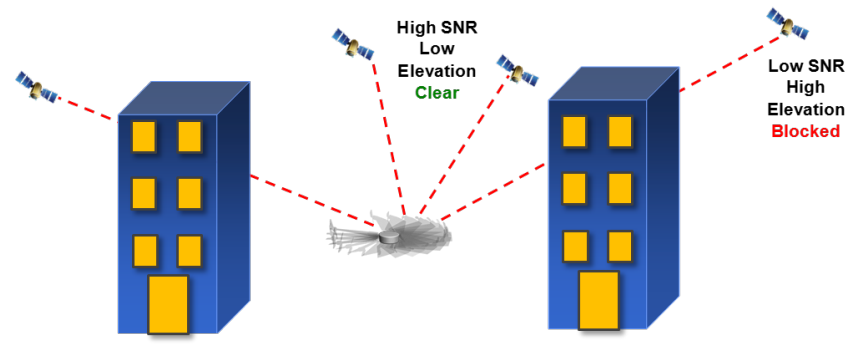

Fig. 1. The location of the specific GPS satellites relative to the MAV as well as their SNR readings are important considerations in determining which directions for the MAV to travel. When a GPS receiver passes too close to a building the signal will be blocked by the obstacle resulting in lower signal-to-noise ratio and degraded localization

street level in urban areas while navigating through tight environments are of great value. These Micro Air Vehicles (MAVs) hold great promise for emerging applications including perimeter security, urban tracking and for making motion pictures.

During these missions, it is crucial for the MAV to maintain an accurate estimate of its position in order to complete the defined objectives. MAVs are typically equipped with a navigation suite consisting of an integrated inertial navigation system (INS) and GPS. Given the low quality INS used on MAVs, the GPS is especially important to keep the INS error bounded as the MAV moves, enabling guidance and control loops to be successfully closed around the vehicle states [2]. Unfortunately, this strong dependence on GPS poses its own unique set of problems for operation in urban environments because GPS signals can become degraded by features such as tall buildings and urban canyons, causing signal outage or non-line-of-sight (NLOS) conditions. If the MAV passes close to a building, some of the satellite signals may become blocked as shown in Figure 1. This blockage of the direct line-of-sight (LOS) leads to multipath signals and large errors in the position estimate, which can have adverse impact on the overall nav solution [3]. To mitigate this, it is important to develop: (a) alternate sources of navigation aiding information; and (b) planning and control algorithms for navigating effectively in GPS-degraded environments which would, for instance, enable the MAV to navigate around obstacles while keeping a clear LOS to the majority of the satellites, thereby minimizing errors in the position estimates.

In this work, we describe a planning and control scheme for a wholly rotating MAV to autonomously fly along routes that maintains high quality of GPS connectivity while track- 
ing an RF source in degraded environments. To maximize RF link strength, we use a greedy algorithm in which the MAV estimates Angle of Arrival (AOA) to the source at each rotation, and then follows the estimated direction until the next rotation is complete. We showed the convergence of this algorithm for the obstacle free case in our previous work [4], while here we consider the case where the RF source is located among buildings in an urban environment. To maintain connectivity to GPS, the MAV detects the direction of nearby buildings via SNR measurements and circumnavigates the obstacles if necessary thus avoiding areas of poor GPS connectivity. This approach offers three unique benefits. First, it offers a low size, weight, power and cost (SWAPC) approach for navigating in the GPS degraded environment because it is based entirely on information which can be decoded by GPS receivers already present on MAVs. It does not require the use of extra hardware such as lidars needed for SLAM-based techniques. Second, it enhances the survivability of the MAV and sustains its mission tempo because it enables the MAV to keep operating, seeking out regions of higher GPS SNR, rather than the current practice of loitering or hovering when GPS quality degrades. Third and perhaps most significant, this technique enables the potential use of the GPS as a low SWAPC long range obstacle avoidance sensor for long-term path planning on MAVs (making up for MAVs' inability to carry bulky and expensive obstacle avoidance sensors such as LIDAR) since paths that improve quality of global positioning are typically obstruction free as well.

Path planning under state estimation uncertainty has been studied in a number of contexts in recent years. A probabilistic navigation method based on the motion estimation uncertainty is presented in [5] that produces paths with tolerable risk of collision with obstacles. A Gaussian augmented Markov decision process method has been shown to be an effective approach to navigating in degraded GPS environments [6]. In [7], Rapidly exploring Random Belief Trees (RRBT) are used to produce paths that avoid unobservable regions of the statespace. There are two key differences between these approaches and the one proposed here. First, our knowledge of the goal position is limited to noisy angle measurements. Second, our low SWAPC package requires extremely computationally efficient solutions to enable realtime operation. Others deal with the position uncertainty associated with degraded GPS in urban environments directly by detecting which GPS signals are affected by multipath or blockage [8], [9], [10], [11]. These solutions often depend on $3 \mathrm{D}$ city models to determine which regions of the environment will provide poor GPS localization. Instead of relying on maps, our approach seeks to determine locally which directions of travel will lead to poor GPS localization, thus greatly reducing the memory requirements.

Source-seeking and localization of RF emitters has been a topic of extensive recent research. In [12], the motion of a mobile robot is utilized to estimate the RSS gradient by taking signal strength measurements at a sequence of locations from an omni-directional antenna. This method depends on the mobile robot to accurately know its position relative to the starting point of the gradient measurement and also relies on monotonicity and symmetry of the signal strength decay as a function of distance between transmitter and receiver which may be problematic in multi-path scenarios. In [13], a rotating directional antenna is mounted on a wheeled mobile robot for the purpose of wireless node localization. The RSS is measured as a function of antenna angle and cross-correlation with a known antenna gain pattern is used to determine the relative bearing between an unknown radio source and the mobile robot. A particle filter is used to determine the location of the unknown radio source. A different approach to source-seeking that uses RSS measurements from an omnidirectional antenna mounted on a UAV to compute the spatial gradient is described in [14]. The problems of frontier exploration and radio sourceseeking were addressed in [15] by using local RSS gradient estimates to govern which frontier waypoint to approach. The problem addressed in [16] is most similar to our application. In that work the I-Bug algorithm, a variant of the Bug algorithms introduced by Lumelsky and Stepanov [17], is used to locate the source of a concave intensity function in the presence of obstacles. Among the above mentioned works, only [15] and [16] consider RF source seeking among obstacles.

The remainder of this manuscript is organized as follows. The Samarai MAV is described in Section II. Section III presents an algorithm suitable for navigating in GPS degraded environments for the application of RF source seeking with noisy angle of arrival measurements. A global recurrence property is shown for this algorithm in Section IV. The results of simulations fed by experimental datasets as well as a live flight experiment are discussed in Section V. Finally, Section VI contains concluding remarks and a discussion of ongoing and future work.

\section{The Samarai Micro Air Vehicle}

The UAS used in this paper is Lockheed Martin's Samarai - a maple seed inspired MAV (see [18], [19], [20] for additional discussion of design, aerodynamic analysis, modeling and control; [21] for flight videos; and [4] for its use for tracking an RF source). The Samarai MAV is a $30 \mathrm{~cm}$ radius single-wing wholly rotating air vehicle capable of vertical takeoff/landing as well as autonomous flight using onboard sensors packaged into an avionics pod, see Figure 2.

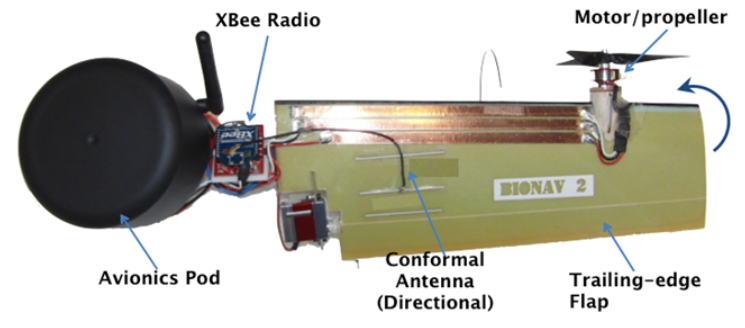

Fig. 2. The Samarai MAV is equipped with an XBee radio and a conformal antenna for seeking out and flying to an RF source as well as an avionics pod that contains a suite of sensors for navigating in the urban environment. 
This vehicle design is simple, stable in hover, aerodynamically efficient and has significantly lower wing loading than conventional rotorcrafts, which reduces its power requirement. The MAV is fitted with an XBee radio with a custom designed directional antenna, and makes RSS and orientation measurements 600 times a second as the MAV rotates and translates. The conformal directional RF antenna on the rotating wing is key towards solving the sourceseeking problem with low complexity: as the antenna rotates, we can measure the signal power incident from different directions. In a Line-of-Sight scenario, the direction in which the RSS is maximal provides the bearing to the source. While this observation forms the basis of our algorithm, in practical settings modifications are necessary. In urban areas where multipath is prevalent the received signal may have significant measurement noise, leading to large errors in the bearing estimate. To reduce such errors, we average the variations in the RSS patterns over multiple rotations, for brevity we refer the reader to [4] for further details.

\section{Algorithm FOR NAVIGATING TO OPTIMIZE GPS QUALITY}

The bearing estimates typically fall into one of two categories: (a) they are roughly correct, with small errors that can be attributed to the bin quantization and small measurement noise or (b) they are outliers and have large errors, either due to large measurement noise or because signals from reflected paths distort the bearing estimate. As in [4] we utilize a firstorder model to capture these possibilities: we assume that reasonably correct estimates occur with probability $1-p$ and outlier estimates occur with probability $p$.

In urban canyons, GPS localization quality can be degraded due to the lack of clear line of sight to a sufficient number of GPS satellites. Our approach to this problem relies on observing the GPS SNR in the direction of each satellite and making inferences about directions of high GPS visibility (allowable) and directions of low GPS visibility (forbidden). The MAV source seeking algorithm uses this information to constrain its path to only go in directions of high GPS visibility.

Each time the GPS receiver reports a position update to the MAV, a triplet of satellite elevations, azimuths, and SNR readings $(\Theta, \Phi, \Sigma)$ are also provided for each satellite detected by the receiver for that measurement. In order to determine which directions are allowed, the algorithm finds the minimum mean squared error solution between a circular step-function and the normalized SNR values as a function of $\Phi$. An example fit can be seen in Figure 3 where directions with a value of 1 (high SNR) are allowable, while directions with a value of 0 (low SNR) indicate the direction of an obstacle and are hence forbidden. While the example in Figure 3 shows SNR data from only 9 satellites, the recent proliferation of satellite system deployments (Galileo, GLONASS, BeiDou) suggests that in the near future GNSS receivers will be capable of decoding signals from far more visible satellites $(>40)$, thereby greatly increasing the resolution of this measurement scheme.

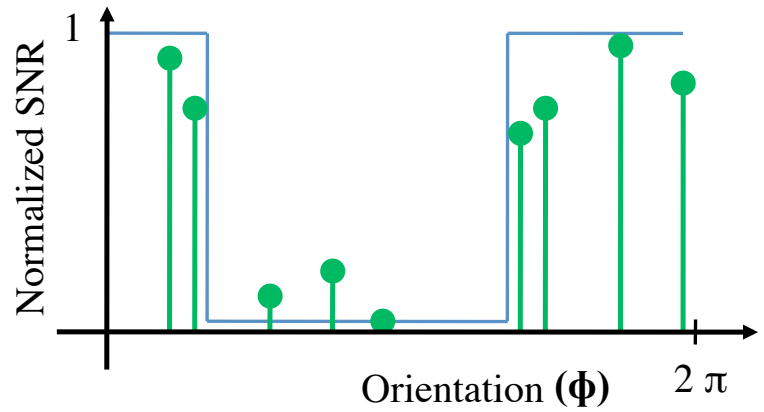

(a) The Minimum Mean Squared Error (MMSE) solution where a value of 1 indicates a clear sky direction, and a value of 0 indicates a direction of an obstacle.

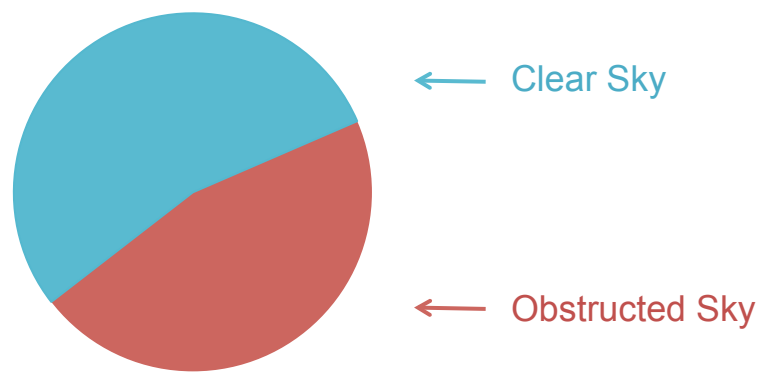

(b) Resulting allowable and forbidden headings for the MAV.

Fig. 3. An illustrative example of how allowable MAV headings are computed from GPS SNR and Azimuth readings.

Let $z=(x, y) \in \mathbb{R}^{2}$ represent the position of the MAV, $q \in\{1,2\}$ denote the mode of the algorithm where $q=1$ represents stochastic source seeking mode and $q=2$ represents the deterministic building avoidance mode. If no nearby buildings are detected, the MAV will operate in a source seeking mode $(q=1)$ according to the algorithm developed in [4]. Suppose that, after the $k$ th rotation, the MAV makes a bearing estimate $\psi_{k}$ of an RF source according to the following model:

$$
\psi_{k}=\left(1-\sigma_{k}\right)\left(\theta_{k}+n_{k}\right)+\sigma_{k} w_{k}, \quad \forall k \in \mathbb{Z}_{\geq 0},
$$

where $\theta_{k}$ denotes the true direction of the source after the $k$ th rotation, $n_{k}$ is an independent and identically distributed (i.i.d.) sequence of additive noise, $w_{k}$ is a sequence of outlier measurements (not necessarily independent), and $\sigma_{k} \in\{0,1\}$ an i.i.d. sequence of Bernoulli random variables with parameter $p \in[0,1)$. The event $\sigma_{k}=1$ corresponds to the sensor producing an outlier measurement after the $k$ th rotation, that is unrelated to the true direction of the source.

Assuming the source is at the origin and the MAV is at $\left(x_{k}, y_{k}\right)$, the true source direction $\theta_{k}$ satisfies

$$
\cos \theta_{k}=-\frac{x_{k}}{\left|z_{k}\right|}, \quad \sin \theta_{k}=-\frac{y_{k}}{\left|z_{k}\right|} .
$$

We assume that at each time step, the MAV moves in the estimated source direction $\psi_{k}$ for a fixed distance $h$. Thus, the MAV's position satisfies the recursive relationship

$$
\begin{aligned}
z_{k+1} & =z_{k}+h\left[\begin{array}{c}
\cos \psi_{k} \\
\sin \psi_{k}
\end{array}\right] \\
& =f\left(1, z_{k}, \psi_{k}\right),
\end{aligned}
$$


where the first argument of $f\left(1, z_{k}, \psi_{k}\right)$ refers to the mode $q=1$.

However, if at any time the MAV detects that it is near a building (using the measurement described in Figure 3), it will switch to building avoidance mode $q=2$. In this mode, we make use of the BUG 2 algorithm from [17]. This algorithm simply takes an acceptable local direction around the building (in the direction of clear sky) until the line in the direction of the last bearing measurement prior to encountering the building is crossed (see Figure 4). To determine this half plane crossing, the MAV needs to remember its position before and after detecting the building. At this point, the MAV moves along this line away from the obstacle until the obstacle is no longer detected at which point $q=1$ and source seeking mode resumes.

\section{Analytical Results}

Let $\mathcal{N} \subset \mathbb{R}^{2}$ be a compact set that represents the building. The distance of the point $z$ to the building is defined as $d(z):=\inf _{y \in \mathcal{N}}|z-y|$. We assume that the MAV detects the building when it is in the region $\mathcal{N}+D \mathbb{B}=\left\{z \in \mathbb{R}^{2}: d(z) \leq\right.$ $D\}$ for some $D>0$. Let $\widehat{D}:=\max _{z_{1}, z_{2} \in \partial(\mathcal{N}+D \mathbb{B})}\left|z_{1}-z_{2}\right|$. The state of the system $(z, q) \in \mathbb{R}^{2} \times\{1,2\}$ and the dynamics are represented by

$$
z_{k+1}=f\left(q_{k}, z_{k}, \psi_{k}\right)
$$

where $\psi \in \mathbb{R}$ is a random input. The mode $q=2$ corresponds to the case where $z \in \mathcal{N}+D \mathbb{B}$ and $q=1$ corresponds to the case where $z \in \mathbb{R}^{2} \backslash(\mathcal{N}+D \mathbb{B})$. We do not assume sufficient separation between the building and target (located at the origin) since the analysis for the algorithm involves establishing recurrence to a neighborhood of the origin instead of asymptotic convergence. Let $(\Omega, \mathcal{F}, \mathbb{P})$ be the underlying probability space. We also exclude the set $\mathcal{N}$ from the set of all possible initial conditions for the MAV.

Assumption 1: The deterministic algorithm used to get around the building will complete in finite time [17], thus it will leave the region $\mathcal{N}+D \mathbb{B}$ without entering $\mathcal{N}$ while preserving the orientation of the MAV to remain sufficiently close (within a small error related to step size) to the direction in which it entered.

We analyze the combined recurrence and building avoidance algorithm by sampling the state $z \in \mathbb{R}^{2}$ at random times and we refer to the sampled state as $\tilde{z}$. To establish recurrence to a neighborhood of the origin, we first prove recurrence properties for the system represented by the sampled state $\tilde{z}$ and then relate it to the original state $z$ under certain technical assumptions. We now consider a modified system with state $\tilde{z}$ by considering the state $z$ only when the state $z$ is outside $\mathcal{N}+D \mathbb{B}$, satisfying

$$
\tilde{z}_{k}=z_{T(k)}
$$

along all sample paths of the state $(z, q)$ where the function $T(k)$ is defined inductively as follows :

$$
\begin{aligned}
T(0) & =0 \\
T(k+1) & =\min \left\{\ell \geq \max \{k+1, T(k)+1\}: q_{\ell}=1\right\} .
\end{aligned}
$$

The dependence of the function $T$ on $\omega \in \Omega$ is suppressed and $T(0)=0$ since we only consider initial conditions for the $\tilde{z}$ system that are outside the set $\mathcal{N}+D \mathbb{B}$.

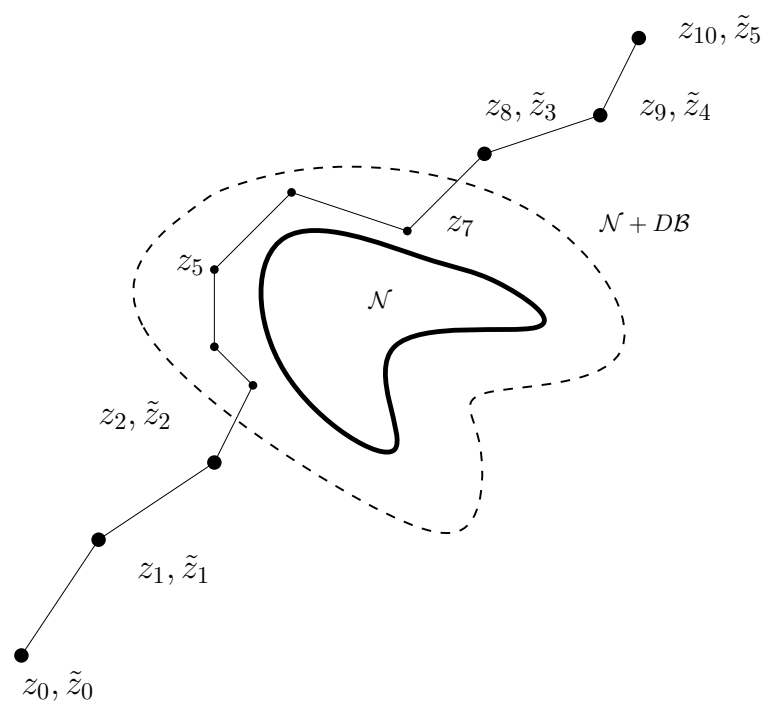

Fig. 4. Sample paths of $z$ and $\tilde{z}$

The sequence $\{\tilde{z}\}_{k \in \mathbb{Z}_{\geq 0}}$ is non-empty for all $k \in \mathbb{Z}_{\geq 0}$ due to properties of the building avoidance algorithm and Assumption 1. From the construction of the state $\tilde{z}$, we observe that $\{\tilde{z}\}_{k \in \mathbb{Z}_{>0}}$ is a subsequence of $\{z\}_{k \in \mathbb{Z}_{>0}}$ and $\tilde{z}_{k} \in \mathbb{R}^{2} \backslash(\mathcal{N}+D \mathbb{B})$ for every $k \in \mathbb{Z}_{\geq 0}$. The dynamics of the state $\tilde{z}$ satisfies

$$
\tilde{z}_{k+1}=\tilde{f}\left(\tilde{z}_{k}, \psi_{k}\right)
$$

where $\tilde{f}(\tilde{z}, \psi)=f(1, \tilde{z}, \psi)$ if $f(1, \tilde{z}, \psi) \in \mathbb{R}^{2} \backslash(\mathcal{N}+D \mathbb{B})$ and $\tilde{f}(\tilde{z}, \psi)=\hat{f}(f(1, \tilde{z}, \psi), \tilde{z})$ if $f(1, \tilde{z}, \psi) \in \mathcal{N}+D \mathbb{B}$. The mapping $\hat{f}:(\mathcal{N}+D \mathbb{B}) \times \mathbb{R}^{2} \backslash(\mathcal{N}+D \mathbb{B}) \rightarrow \mathbb{R}^{2} \backslash(\mathcal{N}+D \mathbb{B})$ can be viewed as the combined action of the deterministic building avoidance algorithm described by the one-step function $f(2, \cdot, \cdot)$ and maps points to a region outside the sensing range of the building.

We now relate the behavior of solutions of the systems (3) and (4) under certain technical assumptions. We impose these assumptions in order to make certain probabilities well defined and to describe the behavior of solutions of systems (3) and (4) in a probabilistic sense. The reader is referred to [22] and [23] for details on random solutions to discrete-time stochastic systems.

Assumption 2: The mapping $\hat{f}:(\mathcal{N}+D \mathbb{B}) \times \mathbb{R}^{2} \backslash(\mathcal{N}+$ $D \mathbb{B}) \rightarrow \mathbb{R}^{2} \backslash(\mathcal{N}+D \mathbb{B})$ is continuous.

Under Assumption 2, unique random solutions exist for the system (4) from all initial conditions $\tilde{z}_{0} \in \mathbb{R}^{2} \backslash(\mathcal{N}+$ $D \mathbb{B})$ and the proof follows the continuity properties of the mapping $f(1, \cdot, \cdot), \hat{f}(\cdot, \cdot)$ and the induction arguments in $[22$, Proposition 4].

We now relate the behavior of solutions of (4) to the solutions of (3) under further technical assumptions which imposes that the solutions of (3) be well defined.

Assumption 3: The mapping $f$ defined in (3) is continuous for $q=2$. 
It follows from (2) that the mapping $(z, \psi) \mapsto f(1, z, \psi)$ is continuous. Then, if Assumption 3 holds, unique random solutions exist for the system (3) from all initial conditions $z_{0} \in \mathbb{R}^{2} \backslash \mathcal{N}$.

Lemma 1: Under Assumptions 1-3, if an open, bounded set $\mathcal{O} \subset \mathbb{R}^{2}$ is globally recurrent for (4) then it is also globally recurrent for (3).

Proof. Since $\mathcal{O} \subset \mathbb{R}^{2}$ is recurrent for (4), it implies that $\mathbb{P}\left(\omega: \exists i \in \mathbb{Z}_{>0}, \tilde{z}_{i}(\omega) \in \mathcal{O}\right)=1 \quad \forall \tilde{z}_{0} \in \mathbb{R}^{2} \backslash(\mathcal{O} \cup$ $(\mathcal{N}+D \mathbb{B}))$. From the construction of (4), it follows that $\mathbb{P}\left(\omega: \exists j \in \mathbb{Z}_{>0}, z_{j}(\omega) \in \mathcal{O}\right)=1$ for all solutions of (3) starting from $z_{0} \in \mathbb{R}^{2} \backslash(\mathcal{O} \cup(\mathcal{N}+D \mathbb{B}))$. In order to establish recurrence to $\mathcal{O}$ from points in the set $(\mathcal{N}+D \mathbb{B}) \backslash \mathcal{N}$ for (3), we note from Assumption 1 that solutions starting from $(\mathcal{N}+D \mathbb{B}) \backslash \mathcal{N}$ reach the set $\mathbb{R}^{2} \backslash(\mathcal{N}+D \mathbb{B})$ surely and hence recurrence of $\mathcal{O}$ follows for (3) from all initial conditions outside the set $\mathcal{N}$.

Assumption 4: Let $\sigma_{k}, \eta_{k}$ and $w_{k}$ be i.i.d random sequences satisfying $\mathbb{E}\left[\cos w_{k}\right]=\mathbb{E}\left[\sin w_{k}\right]=\mathbb{E}\left[\sin \eta_{k}\right]=0$ and $\mathbb{E}\left[\cos \eta_{k}\right]=\eta>0$ for all $k \in \mathbb{Z}_{\geq 0}$.

Theorem 1: Under Assumptions 1-4 the open, bounded set

$$
\mathcal{O}:=\left\{z \in \mathbb{R}^{2}:|z|<\frac{\sqrt{(\widehat{D}+2 h)^{2}+h^{2}}}{2(1-p) \eta}\right\}
$$

is globally recurrent for (3).

Proof. We begin by establishing recurrence of $\mathcal{O}$ for (4) using a proof structure similar to [4, Lemma 1]. Analysis of the sampled state $\tilde{z}$ requires considering two cases with initial conditions constrained to $\mathbb{R}^{2} \backslash(\mathcal{O} \cup(\mathcal{N}+D \mathbb{B}))$. The first case corresponds to the situation when the dynamics for the next step $\tilde{z}_{k+1}$ is represented by $f\left(1, \tilde{z}_{k}, \psi_{k}\right)$ wherein $\tilde{z}_{k+1}$ is at a distance of $h$ from $\tilde{z}_{k}$ in the direction of $\psi_{k}=\left(1-\sigma_{k}\right)\left(\theta_{k}+\eta_{k}\right)+\sigma_{k} w_{k}$. The second case corresponds to the situation when $\tilde{z}_{k+1}=\hat{f}\left(f\left(1, \tilde{z}_{k}, \psi_{k}\right), \tilde{z}_{k}\right)$ wherein $\tilde{z}_{k+1}$ is at a distance of at most $\widehat{D}+2 h$ from $\tilde{z}_{k}$ in the direction of $\psi_{k}=\left(1-\sigma_{k}\right)\left(\theta_{k}+\eta_{k}+\phi\right)+\sigma_{k}\left(w_{k}+\phi\right)$, where upon leaving the region $\mathcal{N}+D \mathbb{B}$ the orientation of the MAV will have a worst-case error of $\phi$ when compared to the orientation when it entered the region. This orientation error of $\phi$ can occur due to the MAV overshooting the half plane by a distance of at most $h$ before completing the building avoidance algorithm, thus

$$
\cos (\phi)=\frac{\widehat{D}+2 h}{\sqrt{(\widehat{D}+2 h)^{2}+h^{2}}} .
$$

Define the Lyapunov function candidate $V(\tilde{z}):=\alpha|\tilde{z}|^{2}$. We now analyze (4) with the worst-case step size of $\widehat{D}+2 h$. It follows from the proof of $[4$, Lemma 1] that

$$
\begin{aligned}
& \mathbb{E}\left[V\left(\tilde{z}_{k+1}\right)-V\left(\tilde{z}_{k}\right) \mid \tilde{z}_{k}\right] \\
& \leq-2 \alpha\left|\tilde{z}_{k}\right|(\widehat{D}+2 h)(1-p) \mathbb{E}\left[\cos \left(\eta_{k}+\phi\right)\right]+\alpha(\widehat{D}+2 h)^{2} \\
& \leq-2 \alpha\left|\tilde{z}_{k}\right|(\widehat{D}+2 h)(1-p) \eta \cos (\phi)+\alpha(\widehat{D}+2 h)^{2} .
\end{aligned}
$$

Setting $\alpha=1 /(\widehat{D}+2 h)^{2}$ we have

$$
\mathbb{E}\left[V\left(\tilde{z}_{k+1}\right)-V\left(\tilde{z}_{k}\right) \mid \tilde{z}_{k}\right] \leq-\rho\left(\left|\tilde{z}_{k}\right|\right)+\mathbb{I}_{[0, \varepsilon)}\left(\left|\tilde{z}_{k}\right|\right)
$$

where $\mathbb{I}_{[0, \varepsilon)}(r)$ is the indicator function taking the value 1 when $0<r<\varepsilon$ and 0 otherwise, $\varepsilon>\frac{\widehat{D}+2 h}{2(1-p) \eta \cos (\phi)}$, and $\rho$ : $\mathbb{R}_{\geq 0} \rightarrow \mathbb{R}_{\geq 0}$ satisfies $\rho(s)=2 s(\widehat{D}+2 h)^{-1}(1-p) \eta \cos (\phi)$ when $s<\varepsilon$ and $\rho(s)=2 s(\widehat{D}+2 h)^{-1}(1-p) \eta \cos (\phi)-1$ otherwise. Since $V$ is radially unbounded and locally bounded, it follows from [24] that $V$ is a Lyapunov function that establishes global recurrence of the set $\mathcal{O}$ for (4). Then, from Lemma 1 it follows that the set $\mathcal{O}$ is recurrent for (3).

\section{NUMERICAL RESUltS AND FLIGHT EXPERIMENTS}

The algorithm described in Section III was validated with numerical simulations in an environment containing a large building developed from experimental data. This algorithm provides the MAV heading commands while its speed and height commands are determined using its own baseline guidance and control system. A large amount of experimental GPS satellite data $(\Theta, \Phi, \Sigma)$ was collected over a large area of free, partially occluded, and fully occluded space. Gross SNR, azimuth, elevation, and satellite ID values were collected for all visible satellites along a lawnmower path surrounding a building, see Figure 5. This information stored and used to produce GPS SNR measurements for simulations of the algorithm described in Section III. An example trajectory of the MAV is shown in Figure 6. Here the MAV(starting at green $\mathrm{X}$ ) is seeking a stationary beacon (shown as red $\mathrm{X}$ ). The MAV starts in source seeking mode $(q=1)$, and as the MAV approaches the building (magenta circle), the latter is detected from a drop in the GPS SNR values. The MAV then switches to operate in building avoidance mode $(q=2)$ until it crosses the half plane (magenta dashed line) defined by the last heading angle before detecting the building. The MAV then switches back to source seeking mode $(q=1)$ (second magenta circle) until the source is reached. The average GPS SNR measurements for this trajectory are shown in Figure 7.

As discussed in Section III, a key attraction of the proposed approach is the fact that it is simple and straightforward to implement onboard the gumstix processor on the MAV. When the source is not located in a maze type corridor, an even simpler form of the algorithm can be used. In the implementation of this algorithm, the MAV goes in the direction of the latest AOA measurement unless that direction is toward obstructed sky and thus forbidden according to Figure 3(b). In this case the MAV turns away from the building to seek out higher SNR satellites. This simplified Bug algorithm avoids the need for memory elements and the resulting paths are shorter due to the MAV only needing to go around the corner of the building before the AOA direction lies within clear sky again. To validate the performance of the algorithm in flight, we carried out experiments in an outdoor environment with virtual structures inserted to represent tall buildings that would interfere with the reception of GPS signals. The presence of these structures had the effect of degrading the quality of signals from all GPS satellites that 


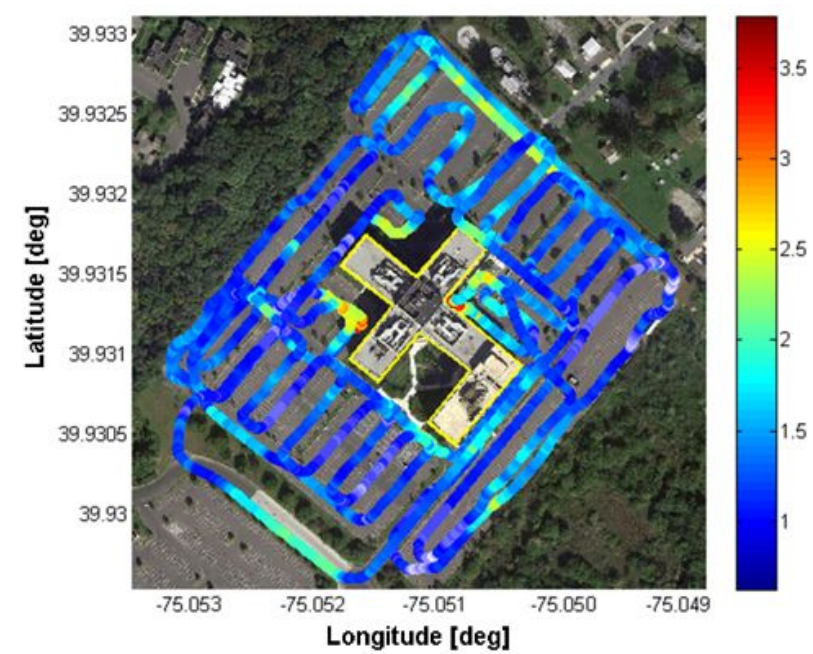

Fig. 5. The numerical simulations were based on experimental data collected by forming a GPS SNR profile of the environment around a building and storing this together with associated azimuth, elevation and satellite ID information to a look-up table. Here the colormap refers to an averaged Horizontal Dilution of Precision (HDOP) measure.

met the occlusion conditions depicted in Figure 1. The goal of the flight experiment was to fly to the RF source along a GPS-Optimal path for this specific environment. Screen captures of the ground control station (GCS) display for the flight experiment are shown in Figure 8.

In Figure 8(a), the algorithm computes a desired heading that corresponds to the closest direction of GPS LOS to the RF line of bearing to the source. This causes the MAV to actually fly at an acute angle to the line of bearing between the start point and the beacon, increasing the number of visible high SNR satellites (Figure 8(b)). As the MAV finally turns to the RF source in Figure 8(c), essentially all the satellites used to compute its nav solution are high SNR satellites, indicating that the MAV showed a preference for a flight path that kept GPS quality high, even though this was not the shortest path to the goal. The MAV's actual flighpath is shown in Figure 9, where the experiment concludes when the MAV reaches the beacon. Because the algorithm seeks out high SNR GPS satellites while keeping the MAV close to the RF-based line of bearing to the beacon, the net effect is that both received signal strength from the RF Beacon and GPS SNR are kept high in this approach (Figure 10 and Figure 11 respectively).

\section{CONCLUDing REMARKS}

In this paper, we have described an algorithm for enabling a MAV to track an RF source in a degraded GPS environment by guiding the MAV along flight paths for which the received signal strength from the RF source is maximized while maintaining a high GPS signal-to-noise ratio. To optimize signal strength to the source, the MAV exploits its rotation to estimate the direction of the signal which it then attempts to follow. To maintain high GPS quality, for all satellites in view we use SNR, azimuth and elevation information to determine desirable directions of travel that avoid areas with

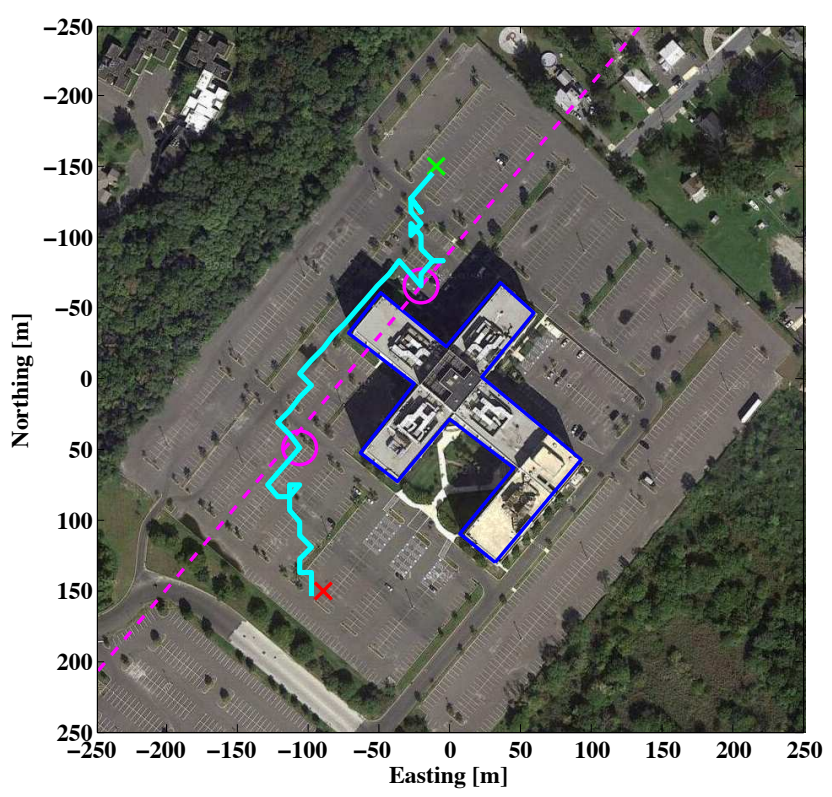

Fig. 6. The MAV locates a stationary RF beacon on the lower left of the building. By flying only along paths of high GPS SNR, rather than solely along the AOA to the beacon, the MAV is able to avoid the building without the use of an explicit obstacle avoidance sensor.

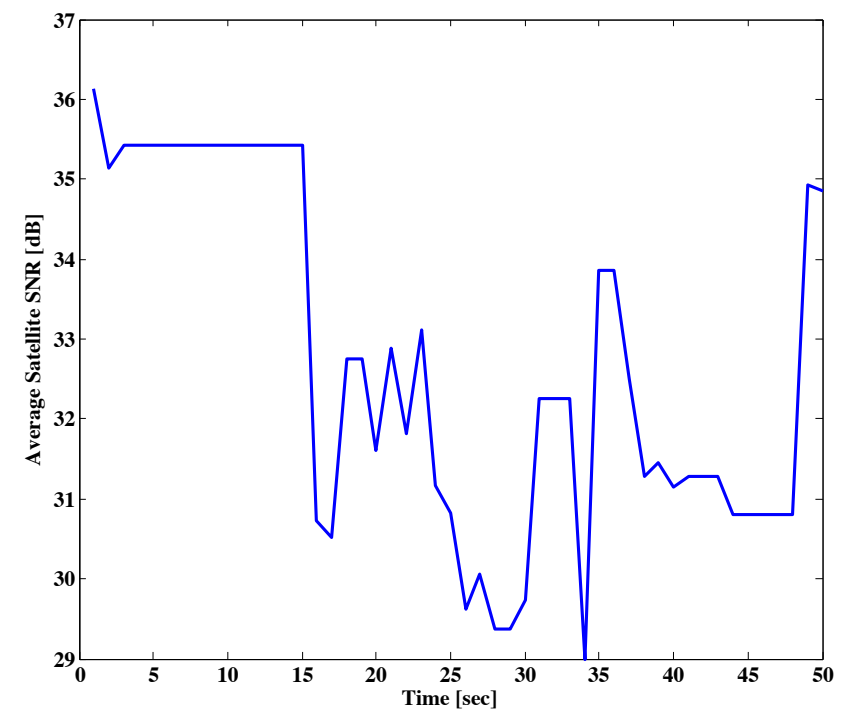

Fig. 7. The variation in average GPS SNR as seen by the GPS receiver on the MAV as it seeks a static RF source.

NLOS conditions. By balancing flight along paths that keep the most number of high SNR satellites visible with the need to fly along the AOA to the source, the MAV continuously improves its view of satellites with the highest SNR while navigating through the environment. Because the approach uses only data that can be extracted by the GPS receivers ubiquitous on almost every MAV, it offers the promise of a low SWAPC approach for enabling MAVs operate in GPS degraded environments as well as a virtual obstacle avoidance sensor for long range path planning on MAVs. The algorithm was successfully implemented onboard a MAV and demonstrated in flight experiments. As future work, we will 
extend the technique to also command the height of the MAV. We will also investigate the use of multiple MAVs as aiding sources for the localization of individuals operating in the environments.

\section{REFERENCES}

[1] K. Fregene, "Unmanned aerial vehicles and control," IEEE Control Systems, vol. 32, no. 5, p. 3234, 2012.

[2] G. A. S. Ahrens, D. Levine and J. How, "Vision-based guidance and control of a hovering vehicle in unknown, GPS-denied environments," in Proc. IEEE International Conference on Robotics and Automation, Kobe, Japan, May 2009, pp. 2643-2648.

[3] J. Kim and S. Sukkarieh, "6DoF SLAM aided GNSS/INS navigation in GNSS denied and unknown environments," Journal of Global Positioning Systems, vol. 4, no. 1-2, p. 120128, 2005.

[4] S. Venkateswaran, J. T. Isaacs, K. Fregene, R. Ratmansky, B. M. Sadler, J. P. Hespanha, and U. Madhow, "RF source-seeking by a micro aerial vehicle using rotation-based angle of arrival estimates," in American Control Conference (ACC), 2013, pp. 2581-2587.

[5] P. Burlina, D. DeMenthon, and L. S. Davis, "Navigation with uncertainty: II. avoiding high collision risk regions," University of Maryland, Tech. Rep., 1991.

[6] P. Lommel, M. W. McConley, and N. Roy, "Robust path planning in GPS-denied environments using the Gaussian augmented markov decision process," http://groups.csail.mit.edu/rrg/papers/EKFAMDP.pdf.

[7] M. W. Achtelik, S. Weiss, M. Chli, and R. Siegwart, "Path planning for motion dependent state estimation on micro aerial vehicles," in IEEE International Conference on Robotics and Automation, 2013.

[8] B. Ben-Moshe, E. Elkin, H. Levi, and A. Weissman, "Improving accuracy of GNSS devices in urban canyons." in Canadian Conference on Computational Geometry (CCCG), 2011.

[9] P. D. Groves, Z. Jiang, L. Wang, and M. K. Ziebart, "Intelligent urban positioning using multi-constellation GNSS with 3D mapping and NLOS signal detection," in Proceedings of the 25th International Technical Meeting of The Satellite Division of the Institute of Navigation (ION GNSS 2012), 2012.

[10] M. Obst, S. Bauer, and G. Wanielik, "Urban multipath detection and mitigation with dynamic 3D maps for reliable land vehicle localization," in IEEE/ION Position Location and Navigation Symposium (PLANS), 2012, pp. 685-691.

[11] S. Bauer, M. Obst, R. Streiter, and G. Wanielik, "Evaluation of shadow maps for non-line-of-sight detection in urban GNSS vehicle localization with VANETsthe GAIN approach," in Proc. of IEEE Intl. Conf. Vehicular Technology, 2013.

[12] K. Dantu, P. Goyal, and G. S. Sukhatme, "Relative bearing estimation from commodity radios," in IEEE International Conference on Robotics and Automation, Kobe, Japan, May 2009, pp. 3215-3221.

[13] J. Graefenstein, A. Albert, P. Biber, and A. Schilling, "Wireless node localization based on RSSI using a rotating antenna on a mobile robot," in Workshop on Positioning, Navigation and Communication, Hanover, Germany, March 2009, pp. 253-259.

[14] A. Wadhwa, U. Madhow, J. Hespanha, and B. Sadler, "Following an RF trail to its source," in 49th Annual Allerton Conference on Communication, Control and Computing, Monticello, IL, October 2011, pp. 580-587.

[15] J. Twigg, J. Fink, P. Yu, and B. Sadler, "RSS gradient-assisted frontier exploration and radio source localization," in International Conference on Robotics and Automation, St. Paul, MN, May 2012, pp. 889-895.

[16] K. Taylor and S. M. LaValle, "I-Bug: An intensity-based bug algorithm," in IEEE International Conference on Robotics and Automation, 2009, pp. 3981-3986.

[17] V. J. Lumelsky and A. A. Stepanov, "Path-planning strategies for a point mobile automaton moving amidst unknown obstacles of arbitrary shape," Algorithmica, vol. 2, no. 1-4, pp. 403-430, 1987.

[18] K. Fregene and C. L. Bolden, "Dynamics and control of a biomimetic single-wing nano air vehicle," in American Control Conference (ACC), 2010, pp. 51-56.

[19] K. Fregene, S. Jameson, D. Sharp, H. Youngren, and D. Stuart, "Development and flight validation of an autonomous mono-wing UAS," in American Helicopter Society Forum, Phoenix, AZ, 2010.

[20] B. Obradovic, K. Fregene, G. Ho, R. Barto, and D. Sharp, "A multi-scale simulation methodology for the samarai monocopter micro UAV," in AIAA Modeling and Simulation Conference, Minneapolis, MN, 2012, pp. 1296-1307.
[21] Lockheed Martin. (2012, Jun.) Samarai - a maple seed inspired UAV. Type the title into the youtube search window to watch the video. [Online]. Available: http://www.youtube.com

[22] S. Grammatico, A. Subbaraman, and A. R. Teel, "Discrete-time stochastic control systems: A continuous Lyapunov function implies robustness to strictly causal perturbations," Automatica, vol. 49, no. 10, pp. 2939-2952, 2013.

[23] A. Teel, "A Matrosov theorem for adversarial markov decision processes," IEEE Transactions on Automatic Control, vol. 58, no. 8, pp. 2142-2148, 2013.

[24] A. R. Teel, J. Hespanha, and A. Subbaraman, "Stochastic difference inclusions: results on recurrence and asymptotic stability in probability," in IEEE 51 st Annual Conference on Decision and Control (CDC), 2012, pp. 4051-4056. 


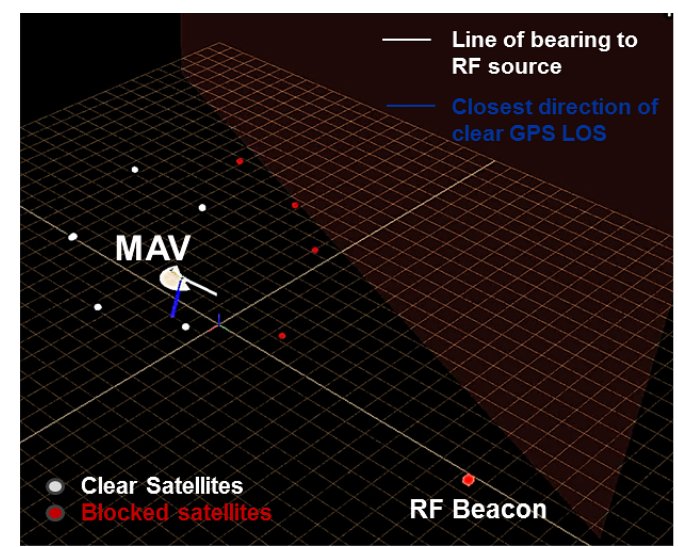

(a) Start of the mission

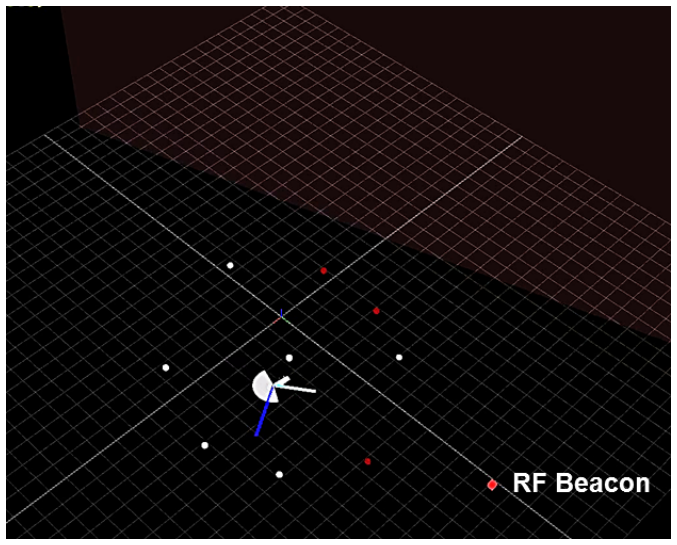

(b) Midway through the mission

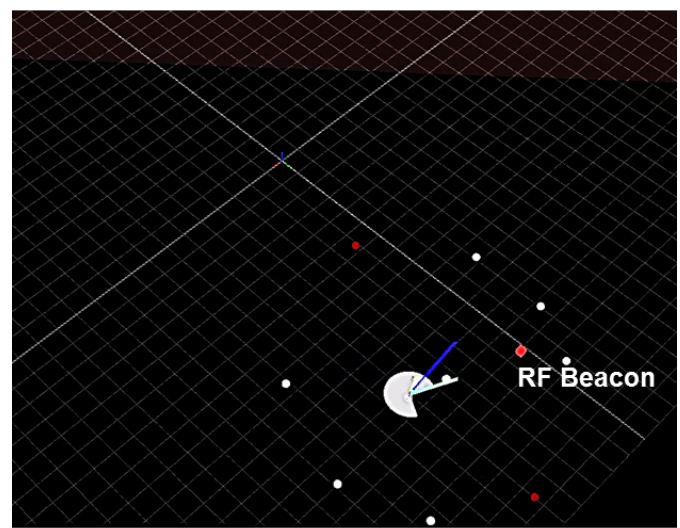

(c) Approaching the beacon

Fig. 8. GCS screen shots of the MAV's Ground Control Station during the flight experiments. At the start of the experiment (a), the GPS satellite configuration is such that line of bearing to the RF source requires the MAV to fly along paths where low SNR satellites (red dots) would be used for nav fix. Midway through the experiment (b), the MAV has flown closer to the RF source along a path that increased LOS to high SNR GPS satellites (white dots). At the end of the experiment (c), the MAV turns to fly to the source after flying a route that kept it away from the structure in the environment. Most satellites used for nav fix at this point have high SNR.

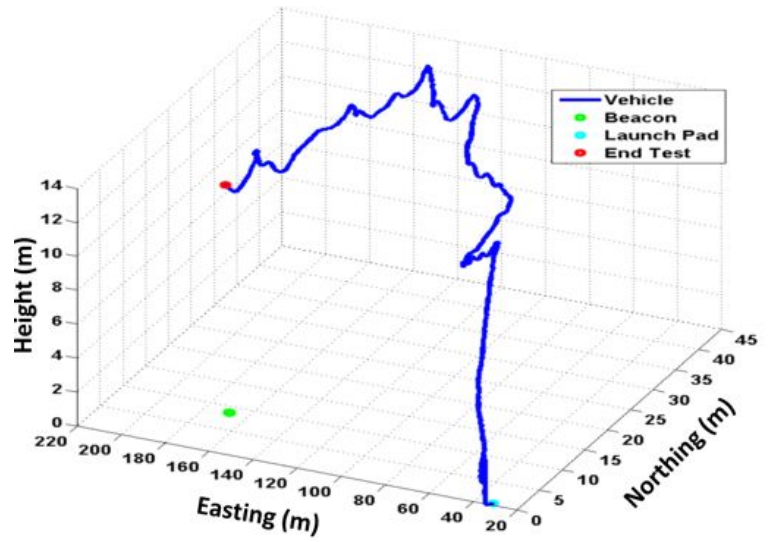

Fig. 9. The MAV's 3D flightpath during the experiment.

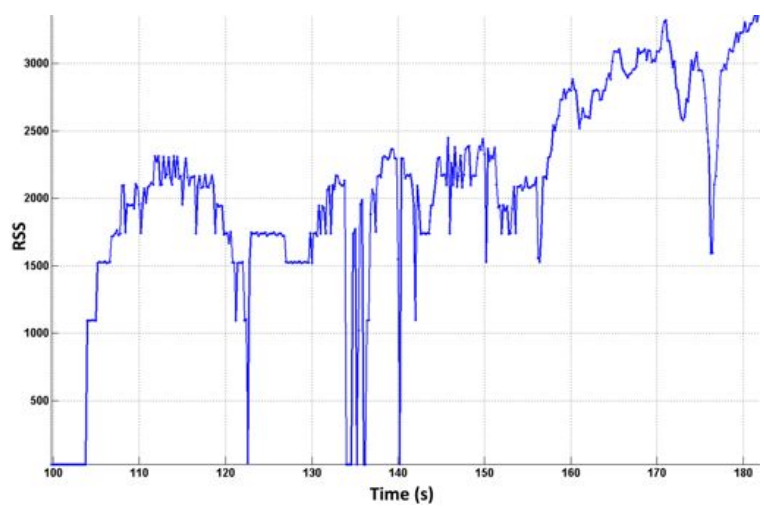

Fig. 10. Variation of RSS from the beacon as the MAV flies in the environment. Apart from a few instances of apparent packet dropout, RSS was high for the majority of the experiment

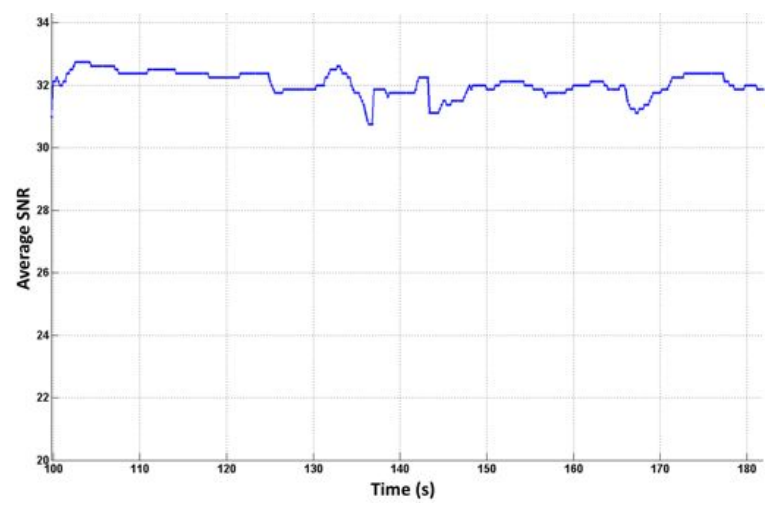

Fig. 11. Average SNR of the satellites used in the MAV's nav fix. While the specific composition of the satellites changed during the flight, the algorithm steered the MAV in directions that enabled the overall SNR to remain high throughout the experiment 